\title{
Evolution in the approach to overlooked ureteral injuries after gynecological surgery
}

\author{
Tomasz Borkowski, Janusz Judycki, Andrzej Borkowski, Maciej Czaplicki, Piotr Radziszewski \\ Department of Urology, Medical University of Warsaw, Poland
}

\begin{abstract}
Objectives:To analyzed the therapeutic results for patients with overlooked iatrogenic ureteral injuries after gynecological surgery, treated at the department since 1990. Before the era of endourology, ureteral injuries were operated on immediately after making a diagnosis. This approach was changed after the popularization of percutaneous nephrostomy (PN) and ureteral stenting using a JJ stent.

Material and methods: 27 patients who were diagnosed with a ureteral injury between the first and sixty-fourth day after injury were included. Only PN was performed in 21 patients (group A). In 6 patients, a JJ stent was introduced either immediately after making a diagnosis or after PN (group B).

Results: In group A, a good therapeutic result was obtained in only 6 patients (28.6\%). Of the 12 patients subjected to PN up to two weeks after injury, 5 had a good result without a need for repair surgery. Of the 9 patients with an injury diagnosed after 3 weeks, only one had a good therapeutic outcome. In Group B, a good result was achieved in 5 out of 6 patients. In 2 patients, a JJ stent was introduced immediately after making the diagnosis, and, in 3 patients, after PN. A successful attempt to "tunnelize" a complete and long obstruction in the sixth patient failed.

Conclusions: Attempting to introduce a JJ stent should be the treatment of choice in patients with an overlooked iatrogenic ureteral injury. If an attempt to introduce the $\mathrm{J} J$ stent fails, PN should be performed as a first step to manage the injury.

Key words: ureteral injury, ureteral stricture, ureteral fistula, percutaneous nephrostomy, JJ stent
\end{abstract}

Ginekologia Polska 2016; 87, 10: 690-696

\section{INTRODUCTION}

Before the era of endourology iatrogenic ureteral injuries diagnosed within a few days after the surgery were treated at the Department of Urology of the Warsaw Medical University by immediate corrective surgery. The operation was carried out through the same incision, and the length of hospital stay was prolonged for only a few days. If the ureteral injury was diagnosed a few or even several weeks after the initial gynaecological operation, only a few centres performed reconstructive surgery immediately at diagnosis. According to the generally accepted opinion that inflammation would make operation more difficult or could have an adverse effect on its result, renal fistula was formed and reconstructive surgery was delayed for several months. It was obviously the worst scenario, because the patient was finally subjected to two additional operations and the pe- riod of recovery increased to several months. This pattern has completely changed with the advent of endourology. Simplicity of primary management and potential avoidance of long-lasting and difficult intervention resulted in total abandoning of early reconstructive operation which was previously the standard of care

In most cases, immediately after making a diagnosis, percutaneous nephrostomy was placed under the ultrasound control or a pig-tail $(J)$ stent was introduced into the kidney under cystoscopy. However, there was a disadvantage of such management because even the patients with a ureter injury who healed spontaneously required several months to recover. Patients who failed initial endoscopic treatment after few weeks still needed subsequent reconstructive operation and prolonged time to final recovery. 


\section{OBJECTIVES}

To retrospectively evaluate results of primary endoscopic management in patients with iatrogenic ureteral injuries treated at the department since 1990 regarding duration of treatment and choosing the most effective approach.

\section{MATERIAL AND METHODS}

From 1990, we have identified 27 patients with unrecognized ureteral injury at the time of various gynaecological surgery. Diagnosis was established between the first and sixty-fourth day after the injury. The patients were divided into 2 groups. Group A included 21 patients subjected to the percutaneous nephrostomy (PN), whereas group B consisted of 6 patients who underwent JJ stent introduction. The type of management, the time that elapsed since the injury, the nature and cause of the injury, the duration of drainage with PN or a JJ stent are summarised in tables 1 and 2 as well as in diagram (Fig. 1).

Group A. Percutaneous nephrostomy was created under the ultrasound control.

The reasons why this procedure was applied in a particular patient instead of ureteral catheterization are difficult to determine, given the retrospective nature of the study. This group also contained a number of patients in whom primary attempt of placing a JJ stent failed after initial difficulties and PN was created.

Inclusion to this group was based on resolution of urine retention or urine leakage through the fistula when only PN was placed with no further attempt to introduce a JJ stent.

Group B. Introduction of a double-folded JJ stent.

The attempts to treat overlooked ureteral injury by introducing a JJ stent were taken less often. We can distinguish two subgroups of patients: I. Originally successful attempts to introduce a JJ stent. II. Initial treatment with PN was followed by introduction of a JJ stent.

\section{RESULTS}

Group A (Precancerous Nephrostomy)

Outcome of the patients treated with PN are shown in Table 1.

In the group of 21 patients initially treated by creating percutaneous nephrostomy, a good result was obtained only in 6 cases (28.6\%). The time that elapsed since the injury to the diagnosis and PN creation seemed to have the greatest impact on success. The chances of healing the ureteral injury after PN decreased with time that elapsed between the injury and the beginning of the treatment. Each day of delay reduces a chance of recovery by $3 \%(R w=1 / 1.03$, $\mathrm{p}<0.12$ ). Among 12 patients subjected to PN up to two weeks after the ureteral injury, 5 obtained a good result and the normal anatomical and functional state was restored without a need for surgery. In 9 patients with an injury diagnosed after 3 weeks (one patient after 19 days), only one case had a good outcome. In 8 remaining cases, the result was poor and patients required subsequent reconstructive surgery. Patients subjected to PN 3 weeks after an injury had almost four times smaller chance of spontaneous ureteral healing $(R w=1 / 3.79, p<0.12)$ than those who underwent PN before the $14^{\text {th }}$ day after the injury. Lack of contrast medium transpassing below the site of obstruction on the initial imaging in patients diagnosed with the injury up to the $14^{\text {th }}$ day seemed to have no impact on the final result. Only 2 patients had incomplete obstruction, 1 treated with good result, the other one failed. Out of 10 patients with complete obstruction, 4 had a good outcome and 6 bad one. In the group of 9 patients with an injury diagnosed

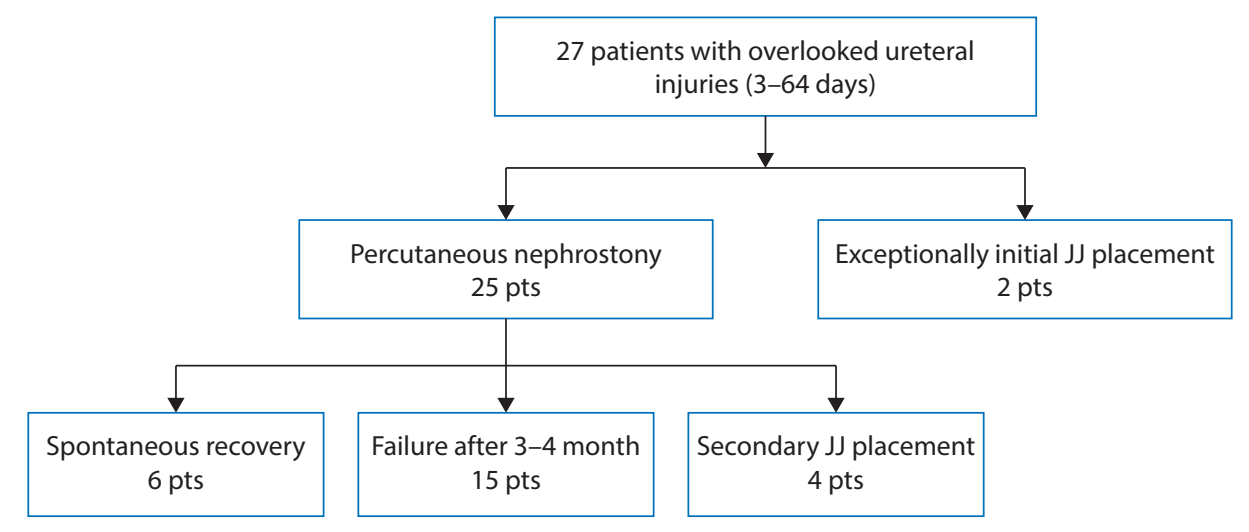

Secondary treatment in case endourological failure:

$11 \times$ open surgical repair

$2 \times$ refusal of treatment

$2 \times$ lost to follow-up

Figure 1. Flowchart of patients with overlooked iatrogenic ureteral injury from both study groups 


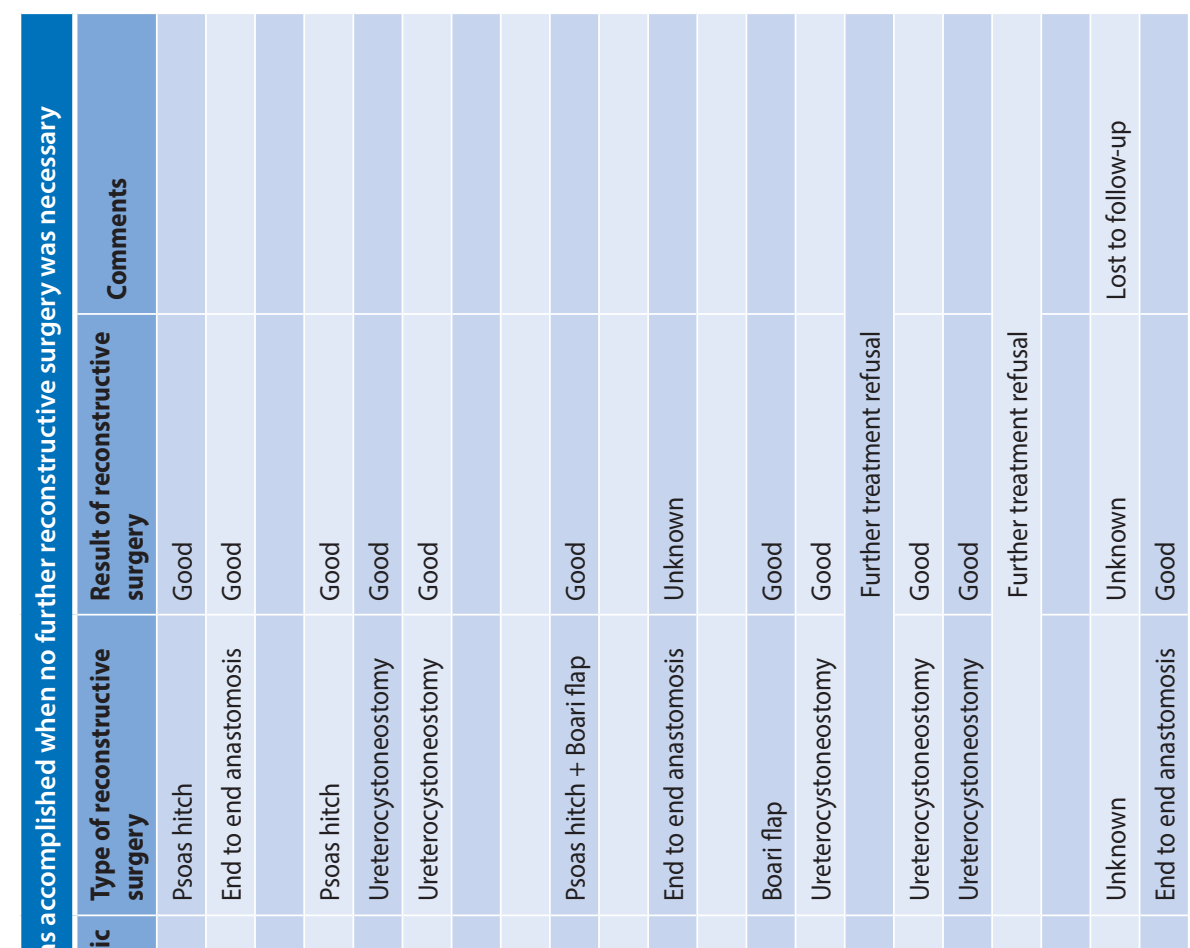

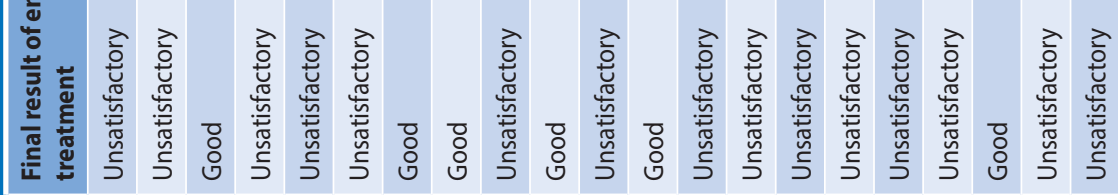

焉蒂

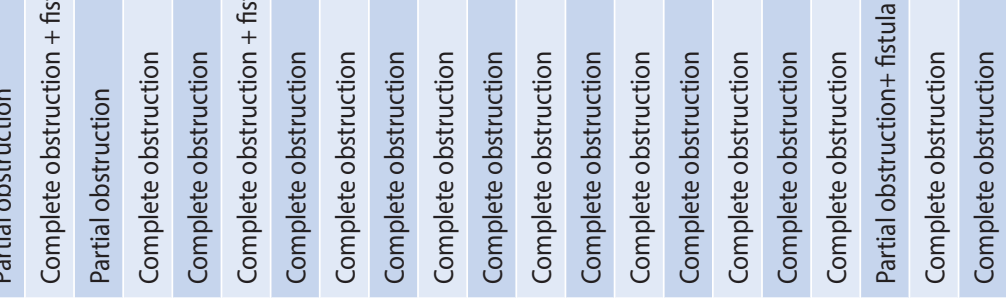

$\bar{n}$

$\frac{\pi_{0}^{\frac{\pi}{0}}}{\sum_{0}}$

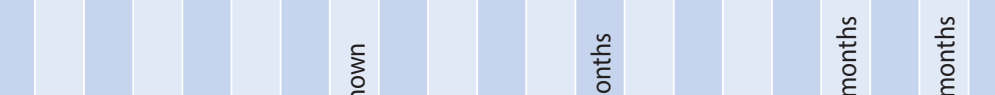

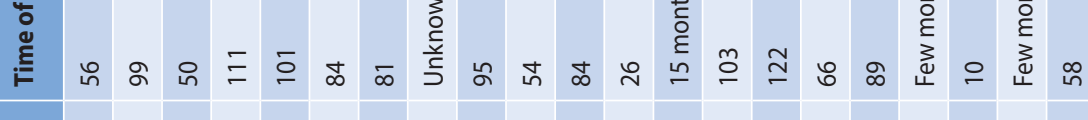

준

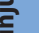

은

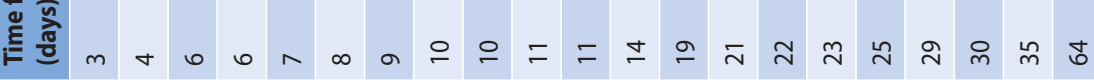

产

选 


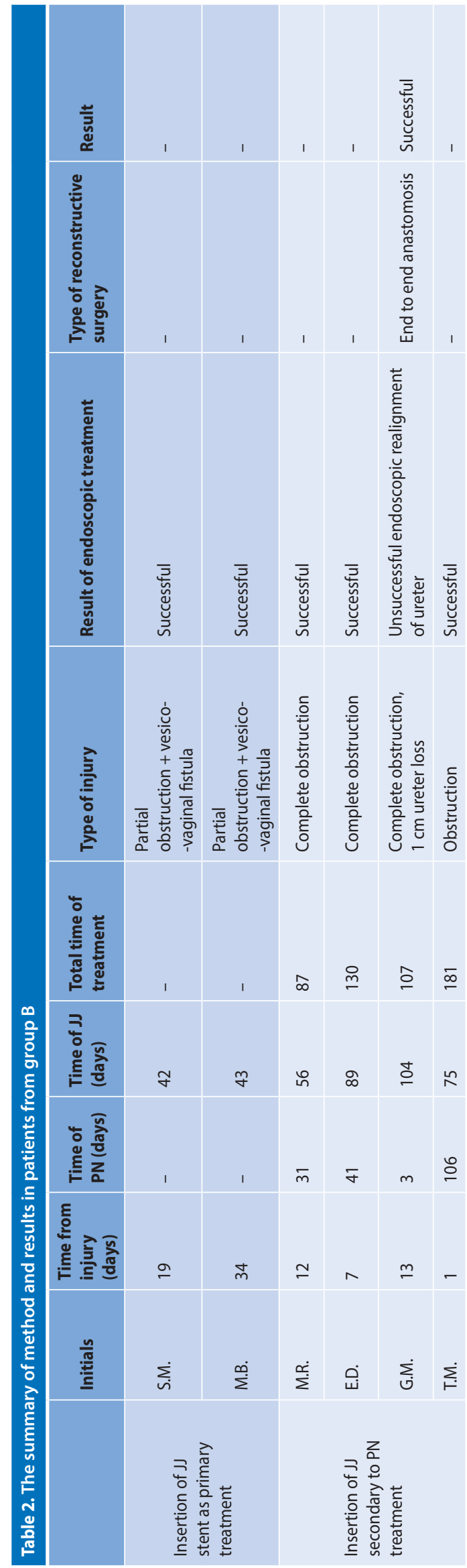

after at least three weeks, in one successful case the obstruction was incomplete and in the 8 unsuccessful cases complete obstruction of the ureter was reported. Therefore, it is most likely that time that elapsed since the diagnosis rather than the nature of injury (within certain limits) has a decisive impact on the outcome, as evidenced by various results obtained in the two subgroups with the same initial injury.

In all cases, the formation of percutaneous nephrostomy protected kidneys from damage, even if final reconstructive operation took place several months later. We were able to determine the results of reconstructive operation in only 11 patients from the group of 15 who didn't recover after PN. The outcome of reconstructive procedures was good: in 5 cases with simple ureterocystoneostomy, in 2 with psoas hitch procedure, in 2 patients with Boari's operation (one of them was combined with the psoas hitch manoeuvre) and in 2 with ureteral end to end anastomosis. We do not know the long term result obtained in one patient subjected to end to end anastomosis; 2 patients refused to undergo the surgery and remained with definitive PN and in 1 patient was lost to follow-up. The influence of patient's age and the type of surgery or injury causing ureteral damage was not analyzed due to the low number of patients in both subgroups.

\section{Group B (insertion of a JJ stent)}

Table 2 presents therapeutic results of the patients from group B.

A JJ stent was introduced immediately after making a diagnosis of ureteral injury only in 2 patients. Figure 2 shows an example of such management. In other 2 cases, a stent was placed after the initial management with PN (for 12 and 7 days). In one patient, a JJ stent was introduced after more than three months of PN when it was decided that the outcome of the previous treatment was unsatisfactory and the resulting narrowing of the ureter should be dilated on a stent. These five patients healed and avoided reconstructive operation. In one patient from this group, the ureteral loss, which was $1 \mathrm{~cm}$ long, did not allow for easy introduction of a stent, so it was merely possible to "tunnelize" obstruction using an ascending route. The result of the treatment was evaluated after keeping JJ stent for 3 months only, but it was disappointing. Due to a small number of patients in this group, it was impossible to analyze an impact of other factors on the result. The statistical analysis of outcome of ureteral injury based on the type of treatment (PN vs. JJ) showed a statistically significant difference $(p<0.0022)$ in the number of positive results in favour of a $\mathrm{JJ}$ stent (Group B).

\section{DISCUSSION}

The preliminary evaluation shows that the management most commonly used at the department failed in 


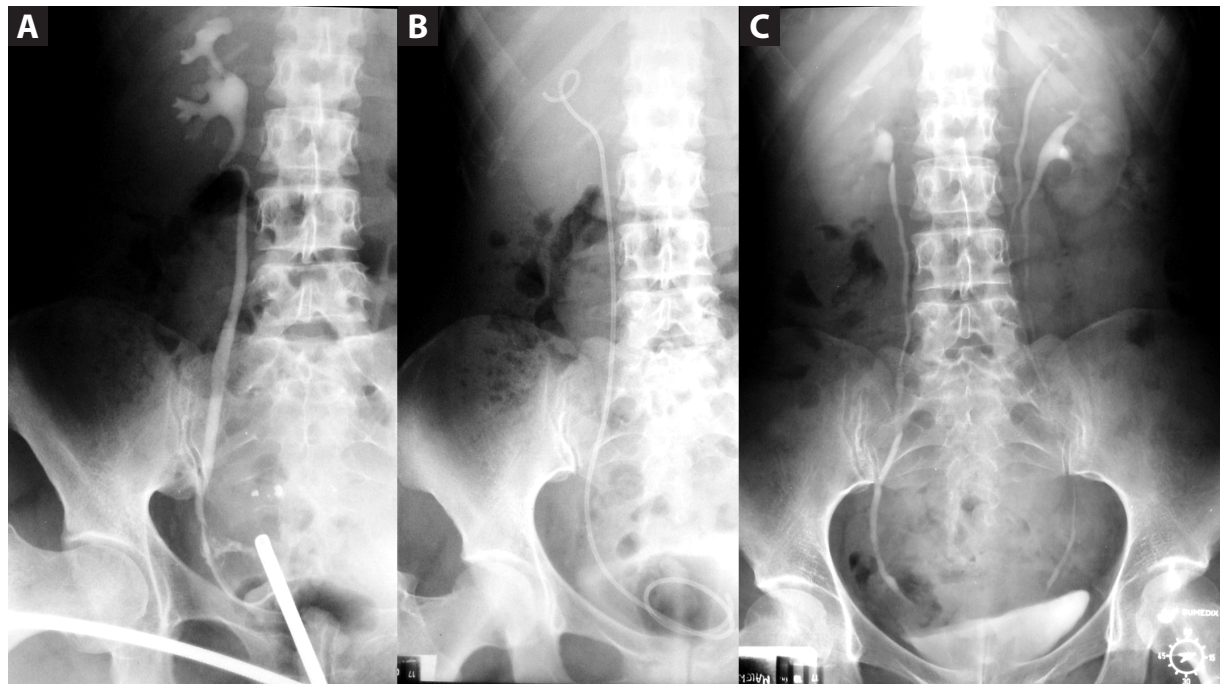

Figure 2. A good result after initial introduction of a JJ stent in a patient with iatrogenic uretero-vaginal fistula and incomplete obstruction: A. Ascending ureteropyelography 34 days after an injury. Visible uretero-vaginal fistula, the contrast agent enters the kidney. B. A JJ stent was held in the ureter for 43 days. C. Urography two months after the removal of a JJ stent

more than $70 \%$ of patients ( 15 out of 21 patients). In more than three quarters of cases it was based on the creation of PN and waiting approximately three months for the result. The reasons why this procedure was applied in a particular patient instead of catheterization are difficult to determine, given the retrospective nature of the study. Based on the literature review of that period, one could draw a conclusion that PN can be an effective procedure and lead to recovery without a need for reconstructive surgery [1-4]. This was also suggested by our own past experiences [5]. It was crucial that in spite of appearances in many patients PN is simpler than catheterization and can be done under local anaesthesia and the ultrasound control. It is also easier to control the position and patency of a stent in PN than a JJ stent. PN was almost always ineffective when an injury was not diagnosed until the end of the second week ( 8 out of 9 patients). This is probably due to the fact that prolonged urine leakage, inflammation and infection, even in a partial injury results in the formation of scar, which leads to complete obstruction. This made recovery by means of PN merely impossible. Introduction of a JJ stent, either immediately after an injury or secondarily was successful in 5 out of 6 patients. In one patient from this group, the ureteral loss, which was $1 \mathrm{~cm}$ long, did not allow for easy introduction of a J Jtent. Finally, we were able to "tunnelize" obstruction using an ascending route. The evaluation of this procedure after 3 months showed disappointing results. Perhaps a stent should have been maintained much longer to allow regeneration of the ureteral wall [6-8].

PN secured the kidney from damage. Eventually in 17 patients completely normal anatomical and functional state was restored, 6 patients healed spontaneously and 11 required reconstructive surgery. In 1 patient the result of surgery is unknown, 1 patient was lost to follow-up and 2 refused surgery. The breakdown proved that the percentage of healing after PN with no reoperation is too small and a 3-4 month period of full recovery is too long. Therefore, the scheme of "endourological treatment" should be changed to the one that would early determine with high probability when the surgery could be avoided. Under these circumstances, longer waiting for the outcome of the treatment is justified. Otherwise, corrective surgery is unavoidable and should be performed as soon as possible. The management of overlooked iatrogenic ureteral injury or ureteral trauma should aim to avoid repair operation, whenever possible or if necessary it ought to be done as soon as possible. Karmouni reported healing of an injury after a successful attempt to introduce a JJ stent in 71\% [9]. Cormio made this effort in 30 patients. It was successful in 16 patients and all of them recovered (100\% of patients), but a stent was kept in place for 3-6 months. All unsuccessful attempts to catheterize were reported in patients more than three weeks after an injury $[10,11]$. Chang cured all patients with uretero-vaginal fistulas who had a possibility to enter a stent, while Toporoff was successful in 5 out of 6 patients $[12,13]$. In the recently published retrospective study conservative management with $J$ J stent was a preferred drainage to obtain spontaneous ureteral healing in 78.5\% [14]. Therefore, an attempt to introduce a JJ stent immediately after making a delayed diagnosis of ureteral injury should be the treatment of choice in most patients and as proven by our material also later than 14 days after an injury. It is not always possible to accomplish this procedure for technical reasons and despite repeated attempts a stent cannot always be introduced above obstruction using an ascending route [15-20]. If an attempt to catheter- 


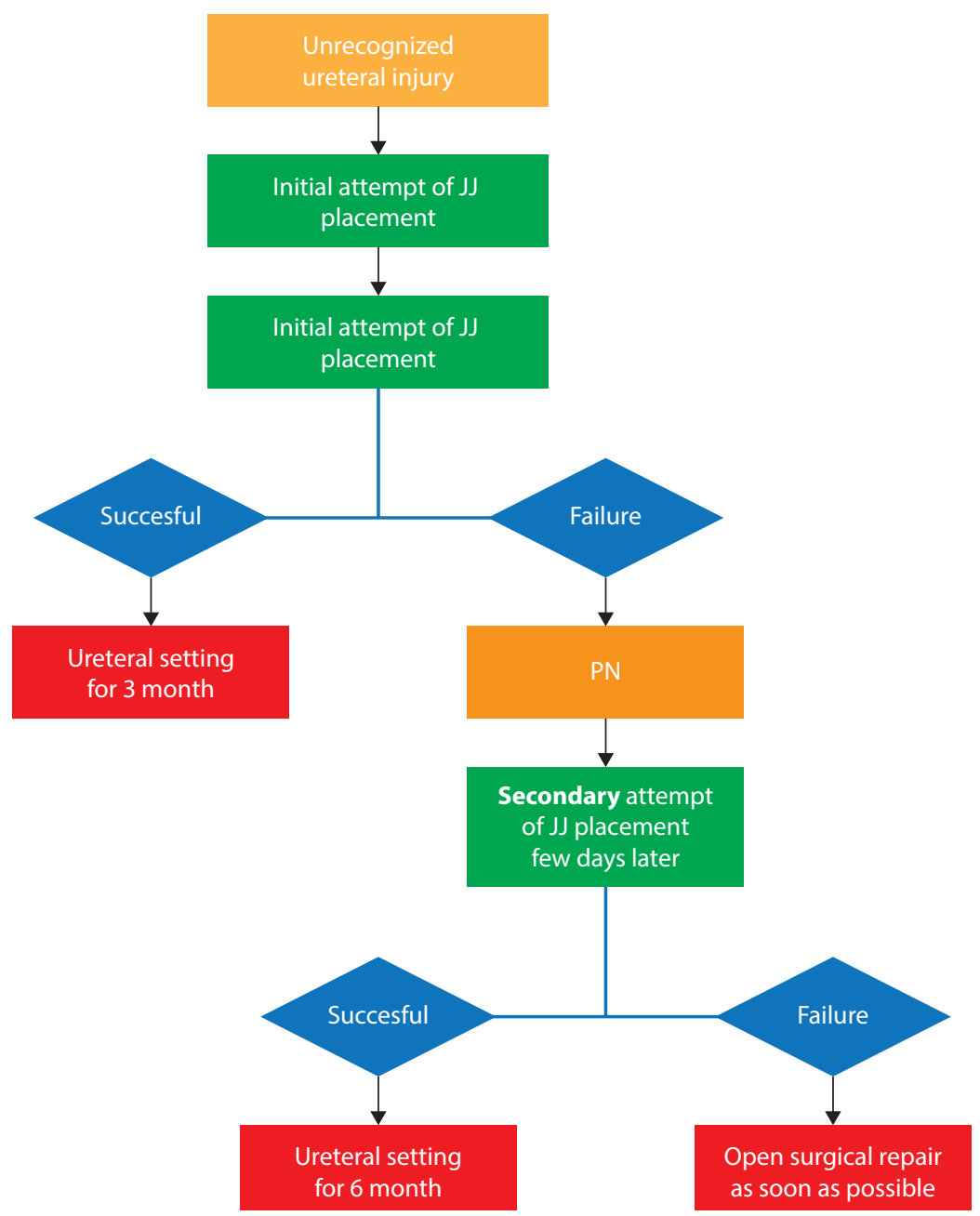

Figure 3. The suggested new algorithm of "endourological" treatment in overlooked iatrogenic ureteral injury

ize failed or it has not been made, the formation of renal fistula is the next or initial step of management. Because PN rarely leads to self-healing, in most patients it should not be regarded as definitive treatment, but as an initial procedure used to make more accurate diagnosis, protect the kidney and possibly decrease or eliminate urine leakage through ureteral fistula. A few days after PN placement, when the symptoms of urine retention in the kidney and above ureter obstruction regress, ascending and descending pyelography should be conducted simultaneously under regional or general anaesthesia. It would allow for more accurate diagnosis than it was possible at the earlier stage and could be helpful for JJ stent re-introduction. If the ureteral loss exceeds $2.0-2.5 \mathrm{~cm}$, there is a little chance of permanent recovery even after "recanalization" or "tunnelization" using two ureterorenoscopes through an ascending and descending route $[11,21]$. These patients should not await reconstructive surgery for several months under the PN cover, but depending on their clinical condition should be operated much sooner if not immediately after mak- ing a diagnosis of ureteral injury. Many reports indicate that such treatment is as effective as postponed surgery $[15,17,18,22-27]$. A prompt reoperation, which couldn't be avoided anyhow in those cases, makes the patient to recover faster. If the imaging studies show short obstruction (a typical ligature or underpinning), an attempt should be made to introduce a JJ stent under the X-ray control. The combine ascending and descending access from the side of PN and the bladder as well as the full range of endourological instruments such as directional wires, special catheters, rigid and flexible ureterorenoscopes might be compulsory to succeed. It can be assumed that in some cases absorbable suture material was degraded to the level that crossing the obstruction with instrument or stent could be achieved. Compared to catheterization done before PN, the manoeuvres are facilitated by reducing local inflammation and straightening ureteral bents above obstruction [28]. If attempts to catheterize repeated after a few days of the PN maintenance fail, they should be reiterated some time later. If the attempts are successful, a double-curved stent 
should be maintained in the ureter for at least 6-12 weeks in short or partial obstruction and easy catheterization and even longer (up to 6 months), if damage is more extensive and after any kind of "tunnelization" [11]. A successful attempt to "tunnelize" a short $1 \mathrm{~cm}$ long defect in our patient G.M. ended unsuccessfully, because it is probably that a stent was kept too short. The patients who fail to be introduced a JJ stent during the initial surgery or later after PN have a bad prognosis of endourological outcome therefore we should consider in these patients an earlier reconstructive surgery whenever it is possible. Postponing it would result in increasing of the duration of treatment. Assessment of time of PN treatment in six of our patient deserves some attention. In these cases mere PN spontaneous resulted in healing of an injury. There is no information about how long PN remained in one patient. In 3 patients it remained for 10 days and in 2 others it was held for 50 and 81 days. However, descending pyelography showed that complete ureteral patency was obtained after 10 and 68 days, respectively. Healing of damage shortly after sole PN proves that damage is not extensive. In these patients, the attempts to catheterize the ureter after a few days since PN would likely lead to success and we would know much sooner that corrective surgery would not be necessary. If the attempts to introduce a JJ stent fail, a persistence of a stent in fistula for three or even four months as in the majority of our patients, especially those diagnosed 3 weeks after an injury and who have complete obstruction results in the prolongation of the treatment and does not prevent from repair operation. Thus, based on the analysis of our results and the review of literature we have created the following algorithm and schedule of management in patients with overlooked iatrogenic ureteral injury and ureteral trauma (Fig. 3).

\section{CONCLUSIONS}

An attempt to introduce a JJ stent should be the initial treatment of choice in patients with overlooked iatrogenic ureteral injury after gynecological surgery. If the attempt is unsuccessful PN should be done through percutaneous puncture and a few days later another attempt should be made to introduce a JJ stent using an ascending and descending route. If catheterization is successful, a stent should be left in the ureter for three months. If the attempt to introduce a JJ stent fails, corrective surgery should be done as soon as possible.

\section{REFERENCES}

1. al-Ali M, Haddad LF. The late treatment of 63 overlooked or complicated ureteral missile injuries: the promise of nephrostomy and role of autotransplantation. J Urol. 1996, 156, 1918-1921.

2. Harshman MW, Pollack HM, Banner MP, [et al.]. Conservative management of ureteral obstruction secondary to suture entrapment. J Urol. $1982,127,121-123$.

3. Lang EK, Lanasa JA, Garrett J, [et al.]. The management of urinary fistulas and strictures with percutaneous ureteral stent catheters. J Urol. 1979, $122,736-740$.

4. Lask D, Abarbanel J, Luttwak Z, [et al.]. Changing trends in the management of iatrogenic ureteral injuries. J Urol. 1995, 154, 1693-1695.

5. Czaplicki M, Malewski AW, Judycki J. Zastosowanie przezskórnej przetoki nerkowej w leczeniu przetoki moczowodowo-pochwowej. Urol Pol. 1993, 46, 71-74.

6. Borkowski A, Kazon M. Regeneration of $2.5 \mathrm{~cm}$ of the ureter. Eur Urol. 1975, 1, 245-247.

7. Cormio L. Ureteric injuries. Clinical and experimental studies. Scand JUrol Nephrol. Suppl. 1995, 171, 1-66.

8. Pastore AL, Palleschi G, Silvestri L, [et al.]. Endoscopic rendezvous procedure for ureteral iatrogenic detachment: report of a case series with long-term outcomes. J Endourol/Endourol Soc. 2015, 29, 415-420.

9. Karmouni T, Patard JJ, Bensalah K, [et al.]. [Urologic management of ureteral iatrogenic lesions]. Prog Urol. 2001, 11, 642-646.

10. Cormio L, Battaglia M, Traficante A, [et al.]. Endourological treatment of ureteric injuries. Br J Urol. 1993, 72, 165-168.

11. Cormio L, Ruutu M, Traficante A, [et al.]. Management of bilateral ureteric injuries after gynaecological and obstetric procedures. Int Urol Nephrol. $1993,25,551-555$.

12. Chang R, Marshall FF, Mitchell S. Percutaneous management of benign ureteral strictures and fistulas. J Urol. 1987, 137, 1126-1131.

13. Toporoff B, Sclafani S, Scalea T, [et al.]. Percutaneous antegrade ureteral stenting as an adjunct for treatment of complicated ureteral injuries. J Trauma. 1992, 32, 534-538.

14. Zilberman DE, Rimon U, Morag R, [et al.]. Non-surgical treatment of latrogenic postoperatively diagnosed ureteral injuries. Isr Med Assoc J. 2015, 17, 227-230.

15. Blandy JP, Badenoch DF, Fowler CG, [et al.]. Early repair of iatrogenic injury to the ureter or bladder after gynecological surgery. JUrol. 1991, 146, 761-765.

16. Dowling RA, Corriere JN, Jr., Sandler CM. latrogenic ureteral injury. J Urol. 1986, 135, 912-915.

17. Ghali AM, El Malik EM, Ibrahim Al, [et al.]. Ureteric injuries: diagnosis, management, and outcome. J Trauma. 1999, 46, 150-158.

18. Hoch WH, Kursh ED, Persky L. Early, aggressive management of intraoperative ureteral injuries. J Urol. 1975, 114, 530-532.

19. Oh BR, Kwon DD, Park KS, [et al.]. Late presentation of ureteral injury after laparoscopic surgery. Obstet Gynecol. 2000, 95, 337-339.

20. Sakellariou P, Protopapas AG, Voulgaris Z, [et al.]. Management of ureteric injuries during gynecological operations: 10 years experience. Eur J Obstet Gynecol Reprod Biol. 2002, 101, 179-184.

21. Liu $C$, Zhang $X$, Xue D, [et al.]. Endoscopic realignment in the management of complete transected ureter. Int Urol Nephrol. 2014, 46, 335-340.

22. Flynn JT, Tiptaft RC, Woodhouse CR, [et al.]. The early and aggressive repair of iatrogenic ureteric injuries. Br J Urol. 1979, 51, 454-457.

23. Soong Y, Lim PH. Urological injuries in gynaecological practice-when is the optimal time for repair? Singapore Med J. 1997, 38, 475-478.

24. Badenoch DF, Tiptaft RC, Thakar DR, [et al.]. Early repair of accidental injury to the ureter or bladder following gynaecological surgery. $\mathrm{Br}$ J Urol. 1987, 59, 516-518.

25. Witters $S$, Cornelissen $M$, Vereecken R. latrogenic ureteral injury: aggressive or conservative treatment. Am J Obstet Gynecol. 1986, 155, 582-584.

26. Beland $G$. Early treatment of ureteral injuries found after gynecological surgery. J Urol. 1977, 118, 25-27.

27. St Lezin MA, Stoller ML. Surgical ureteral injuries. Urology. 1991, 38, 497-506.

28. Razvi HA, Denstedt JD. Endoscopic management of ureteral injury after cesarean section. J Endourol. 1994, 8, 345-347. 